\title{
ON IMPLICIT ACTIVE CONSTRAINTS IN LINEAR SEMI-INFINITE PROGRAMS WITH UNBOUNDED COEFFICIENTS
}

\author{
M.A. GOBERNA, G. A. LANCHO, M.I. TODOROV, AND V.N. VERA DE SERIO
}

\begin{abstract}
The concept of implicit active constraints at a given point provides useful local information about the solution set of linear semi-infinite systems and about the optimal set in linear semi-infinite programming provided the set of gradient vectors of the constraints is bounded, commonly under the additional assumption that there exists some strong Slater point. This paper shows that the mentioned global boundedness condition can be replaced by a weaker local condition (LUB) based on locally active constraints (active in a ball of small radius whose center is some nominal point), providing geometric information about the solution set and Karush-Kuhn-Tucker type conditions for the optimal solution to be strongly unique. The maintaining of the latter property under sufficiently small perturbations of all the data is also analyzed, giving a characterization of its stability with respect to these perturbations in terms of the strong Slater condition, the so-called Extended-Nürnberger condition, and the LUB condition.
\end{abstract}

\section{INTRODUCTION}

We consider given a consistent linear semi-infinite programming (LSIP) problem

$$
P: \operatorname{Inf} c^{\prime} x \text { s.t. } a_{t}^{\prime} x \geq b_{t}, t \in T,
$$

where $c \in \mathbb{R}^{n}, T$ is an arbitrary infinite index set, and $a .: T \rightarrow \mathbb{R}^{n}$ and $b .: T \rightarrow$ $\mathbb{R}$ are arbitrary functions. We denote by $\sigma=\left\{a_{t}^{\prime} x \geq b_{t}, t \in T\right\}, F$, and $F^{*}$ the constraint system, the feasible set, and the optimal set of $P$, respectively. Observe that $F$ can also be represented as $\left\{x \in \mathbb{R}^{n}: f(x) \leq 0\right\}$, where $f$ is the supremum function of $\sigma$, i.e., the lower semicontinuous convex function $f: \mathbb{R}^{n} \rightarrow \mathbb{R} \cup\{+\infty\}$ such that $f(x):=\sup _{t \in T}\left(b_{t}-a_{t}^{\prime} x\right)$. The system $\sigma$ is said to satisfy the strong Slater (SS in brief) condition if the convex system $\{f(x) \leq 0\}$ satisfies the Slater condition $\inf _{x \in F} f(x)<0$. An optimal solution $\bar{x} \in F^{*}$ is strongly unique if there exists $\alpha>0$ such that

$$
c^{\prime} x \geq c^{\prime} \bar{x}+\alpha\|x-\bar{x}\|
$$

for all $x \in F$. Here, $\|\cdot\|$ stands for the euclidean norm.

The system $\sigma$ is said to be upper bounded (UB in brief) if the set of left-hand-side vectors, $\left\{a_{t}: t \in T\right\}$, is bounded. It is known that the global upper boundedness of these vectors allows to obtain valuable information about the geometry of the feasible set $F$, and also about the (strong) uniqueness of solutions of $P$. The purpose of this paper is to extend these results to a large class of LSIP problems by replacing the UB condition by a weaker one whose definition involves the intuitive concept of $\gamma$-active constraint: given $\gamma>0$, the inequality $a_{t}^{\prime} x \geq b_{t}$ is a $\gamma$-active constraint at $\bar{x} \in F$ if there exists $y \in B(\bar{x}, \gamma)$ (the open ball centered at $\bar{x}$ with radius $\gamma$ )

Date: May 4, 2010.

1991 Mathematics Subject Classification. 90C05, 90C34, 15A39, 90C31.

Key words and phrases. linear semi-infinite programming, implicit active constraints, extended active constraints, locally upper bounded systems, strongly unique solution, stability. 
such that $a_{t}^{\prime} y=b_{t}$. The set of left-hand-side vectors of the $\gamma$-active constraints at $\bar{x}$ is denoted by $W(\bar{x}, \gamma)$ :

$$
W(\bar{x}, \gamma)=\left\{a_{t}: a_{t}^{\prime} \bar{x}=b_{t} \text { for some } y \in B(\bar{x}, \gamma)\right\} .
$$

We say that $\sigma$ is locally upper bounded (LUB in short) at $\bar{x} \in F$ if there exists $\gamma>0$ such that $W(\bar{x}, \gamma)$ is bounded (this concept appeared by the first time, as an assumption, in [9, Proposition 3]). We are primarily interested in those LSIP problems whose constraint system is not UB but it is at least LUB at some feasible point. This local condition combined with the strong Slater condition allows us to study some geometric properties of $F$, and to provide optimality and strongly unique optimality tests of Karush-Kuhn-Tucker type. Moreover, with the aid of the Extended-Nürnberger condition (see its definition in Section 5), we characterize the stability, with respect to data perturbations, of linear semi-infinite problems which have a strong unique optimal solution (Theorem 3, in Section 5).

In certain LSIP applications, the user would like to determine from the data, the triple $(a, b, c)$, whether $F$ is full dimensional or not and, in the first case, might want to classify a given feasible point $\bar{x}$ as an interior or a boundary point of $F$. In the latter case, the practitioners would like to know, by means of a Karush-KuhnTucker type condition, whether a given boundary point $\bar{x}$ is an element of $F^{*}$ or not, even more whether it is a unique optimal solution, or a strongly unique optimal solution. The existence of a unique optimal solution is a requirement for wellposedness in Tychonov'sense ([4], [13]), whereas the existence of a strongly unique optimal solution guarantees the computational efficiency of certain LSIP numerical methods ([12]) and it is actually equivalent to the metric regularity of the inverse of the optimal set mapping for an important class of LSIP problems ([3], where a characterization of strongly unique optimal solutions due to Nürnberger ([14]) plays a crucial role). On the other hand, in many practical situations, the triple $(a, b, c) \in\left(\mathbb{R}^{n}\right)^{T} \times \mathbb{R}^{T} \times \mathbb{R}^{n}$ (called the nominal data in the stability framework) can be perturbed due to either measurement errors or rounding errors occurring during the computation process. Then, in the favorable case that $P$ has a strongly unique optimal solution, it can be interesting to know whether or not this property is preserved by sufficiently small perturbations of the data, whose size is measured through the pseudometric of the uniform convergence defined in (5.1). Observe that the existence of a strongly unique optimal solution depends on $F$ and $c$ whereas the maintaining or not of this desirable property under sufficiently small perturbations depends on the nominal data $(a, b, c)$.

All the questions in the above paragraph can be easily answered when $T$ is finite (ordinary linear programming) by means of the set of active constraints at $\bar{x},\left\{a_{t}: t \in T(\bar{x})\right\}$, where $T(\bar{x}):=\left\{t \in T: a_{t}^{\prime} \bar{x}=b_{t}\right\}$ is the set of active indexes at $\bar{x}$. For an infinite set $T$, it is already known how to answer these questions when $\sigma$ is UB, just replacing the set of active constraints by the so-called set of implicit active constraints at $\bar{x}$,

$$
A(\bar{x}):=\left\{a \in \mathbb{R}^{n}:\left(\begin{array}{c}
a \\
a^{\prime} \bar{x}
\end{array}\right) \in \operatorname{cl} D\right\}
$$

where

$$
D:=\left\{\left(\begin{array}{c}
a_{t} \\
b_{t}
\end{array}\right), t \in T\right\}
$$

is the set of coefficients of $\sigma$. This concept was introduced in [11] and the given name recalls the fact that $a \in A(\bar{x})$ implies $a^{\prime} x \geq a^{\prime} \bar{x}$ for all $x \in F$ by the nonhomogeneous Farkas lemma. Clearly, $A(\bar{x})$ is a closed subset of $\mathbb{R}^{n}$ such that $\left\{a_{t}, t \in T(\bar{x})\right\} \subset A(\bar{x})$, where this inclusion can be stricted even for UB systems 
(see Example 5 in Section 3). As shown in [8], [9], and [11], most results on LSIP problems with UB constraint system require the SS property of $\sigma$, which is equivalent to the existence of $\widehat{x} \in \mathbb{R}^{n}$ and $\varepsilon>0$ such that $a_{t}^{\prime} \widehat{x} \geq b_{t}+\varepsilon$ for all $t \in T$. In such a case $\widehat{x}$ is said to be an $S S$ point of $\sigma$; a Slater point satisfies $a_{t}^{\prime} x>b_{t}$ for all $t \in T$. The SS condition can be seen as a constraint qualification stronger than the Slater one, and also as a stability condition, as far as it is equivalent to assert the maintaining of the consistency of the constraint system under sufficiently small perturbations of $a$ and $b$ ([7, Theorem 3.1]).

Let us fix the notation we use in this paper. Given a subset $X$ of some topological space, int $X, \operatorname{cl} X$ and bd $X$ represent the interior, the closure, and the boundary of $X$, respectively. Given a non-empty set $X \subset \mathbb{R}^{n}$, equipped with the Euclidean norm $\|\cdot\|$, by conv $X$, cone $X$, and $\operatorname{dim} X$ we denote the convex hull, the convex conical hull, and the dimension of $X$, respectively. Moreover, we define cone $\emptyset=\left\{0_{n}\right\}$ and denote by $X^{0}$ the positive polar of a given convex cone $X$. Given $\bar{x} \in X$, where $X$ is a convex set, we denote with $D(X, \bar{x})$ the cone of feasible directions at $\bar{x}$. Given $x, y \in \mathbb{R}^{n},[x, y]=\{(1-\alpha) x+\alpha y: \alpha \in[0,1]\}$ (and similar definitions for $\left.] x, y\right]$, etc.); the null-vector will be denoted by $0_{n}$. All the vectors in the finite dimensional spaces are column vectors; but we use indistinctly $(a, b)$ and $\left(\begin{array}{l}a \\ b\end{array}\right), a \in \mathbb{R}^{n}, b \in \mathbb{R}$, for notational convenience. Finally, $\lim _{k} x_{k}=x$ and $x_{k} \rightarrow x$ should be interpreted as $\lim _{k \rightarrow \infty} x_{k}=x$.

The paper is organized as follows. Section 2 shows that the finiteness of the supremum function $f$ is a transition condition between the UB condition and the LUB property at every feasible point. Concerning $f$, observe that computing $f(x)$, $x \in \mathbb{R}^{n}$, requires to solve a global optimization problem on $T$ (a hard task in general). Moreover, in the case that an explicit expression of $f$ is available, the amount of information on $F$ and $P$ obtainable from $f$ is very limited, even though the UB property holds, in particular the information relative to the perturbations of the data (this approach was already explored in [9]). For this reason $f$ plays a very restricted role in this theory. In Section 3, we characterize $A(\bar{x})$ assuming that $\sigma$ is LUB at $\bar{x}$. Although all the results there can be directly proved, it is preferable to re-scale $\sigma$ in order to apply the known theory for UB systems; Section 4 gives some geometric results about $F$. Section 5 determines whether a given $\bar{x} \in F$ is the (strongly) unique solution of $P$ or not by means of a Karush-Kuhn-Tucker condition under the assumption that $\sigma$ is LUB at $\bar{x}$. It also discusses the stability of the strong uniqueness property under small data perturbations, which obviously requires the SS property.

\section{The SUPREMUM FUNCTION}

In LSIP it is not always the case that an SS element is an interior point of the solution set $F$. The following proposition analyzes this situation in relation with the supremum function $f$.

Proposition 1. If $\sigma$ is $S S$, then $F$ is the closure of the set of SS elements of $\sigma$. If, additionally, $f$ is finite-valued, then int $F$ is the set of SS elements.

Proof: Since $f$ is a lower semicontinuous, proper and convex function such that $\inf _{x \in \mathbb{R}^{n}} f(x)<0$ (because $\sigma$ is SS) one has, by [15, Theorem 7.6],

$$
F=\left\{x \in \mathbb{R}^{n}: f(x) \leq 0\right\}=\operatorname{cl}\left\{x \in \mathbb{R}^{n}: f(x)<0\right\} .
$$

If $f$ is finite-valued, then $f: \mathbb{R}^{n} \rightarrow \mathbb{R}$ is continuous and, from [15, Theorem 7.6], we get

$$
\operatorname{int} F=\left\{x \in \mathbb{R}^{n}: f(x)<0\right\}
$$


The following property shows that the finiteness of the supremum function $f$ is a transition condition between the UB condition and the LUB property at every feasible point.

Proposition 2. (i) If $\sigma$ is $U B$, then $f$ is finite-valued.

(ii) If $f$ is finite-valued, then $\sigma$ is LUB at every $x \in F$.

Proof: (i) Assume that $\sigma$ is UB and take an arbitrary $\bar{x} \in F$. Then, given $x \in \mathbb{R}^{n}$, we have

$$
\begin{aligned}
f(x) & =\sup _{t \in T}\left(b_{t}-a_{t}^{\prime} x\right) \leq \sup _{t \in T} a_{t}^{\prime}(\bar{x}-x) \\
& \leq\|\bar{x}-x\| \sup _{t \in T}\left\{\left\|a_{t}\right\|, t \in T\right\}<+\infty
\end{aligned}
$$

(ii) Now, suppose that $f$ is finite-valued, i.e., $\operatorname{dom} f=\mathbb{R}^{n}$. If $F=\mathbb{R}^{n}$, then $\left\{a_{t}, t \in T\right\}=\left\{0_{n}\right\}$ and $\sigma$ is trivially UB. Thus we assume that $F \neq \mathbb{R}^{n}$. We prove now that $\sigma$ is LUB at any $x \in F$ discussing the two possible cases for the position of $x$ relative to $F$ :

If $x \in \operatorname{bd} F$, then $\sigma$ is LUB at $x$ by [9, Proposition 1] because int $\operatorname{dom} f=\mathbb{R}^{n}$.

Otherwise, if $x \in \operatorname{int} F$, taking $\gamma>0$ such that $B(x, \gamma) \subset F$ we have $W(x, \gamma) \subset$ $\left\{0_{n}\right\}$, so that $\sigma$ is also LUB at $x$.

The next examples show that the converse statements of (i) and (ii) in Proposition 2 fail even assuming that either $F$ is a singleton or $\sigma$ is SS. To find $W(\bar{x}, \gamma)$, notice the following useful representation

$$
W(\bar{x}, \gamma) \backslash\left\{0_{n}\right\}=\left\{a_{t} \neq 0_{n}: a_{t}^{\prime} \bar{x}-b_{t}<\gamma\left\|a_{t}\right\|\right\} .
$$

Example 1. $f$ finite-valued and $\sigma$ not $U B$ : Let $n=1$ and $\sigma=\left\{t x \geq-t^{2}, t \in \mathbb{R}\right\}$. Here $\left\{a_{t}, t \in \mathbb{R}\right\}=\mathbb{R}$ whereas $f(x)=\frac{x^{2}}{4}$ is finite-valued. Observe that, in this case, $F=\{0\}$ and $W(0, \gamma)=]-\gamma, \gamma[$ for all $\gamma>0$, i.e. all the sets of $\gamma$-active constraints at 0 are bounded.

Example 2. $\sigma$ LUB at the unique feasible point, $f$ not finite-valued: Let $n=1$ and consider the following representation of $F=\{0\}$ :

$$
\sigma=\left\{-\frac{2 t}{\left(1-t^{2}\right)^{2}} x \geq-\frac{t^{4}+t^{2}}{\left(1-t^{2}\right)^{2}}, t \in\right]-1,1[\} .
$$

Its supremum function

$$
f(x)=\left\{\begin{array}{cl}
\frac{x^{2}}{1-x^{2}}, & x \in]-1,1[ \\
+\infty, & \text { otherwise, }
\end{array}\right.
$$

is not finite-valued. On the other hand,

$$
\left.W\left(0, \frac{5}{16}\right)=\left\{-\frac{2 t}{\left(1-t^{2}\right)^{2}}, t \in\right]-\frac{1}{2}, \frac{1}{2}[\}=\right]-\frac{16}{9}, \frac{16}{9}[,
$$

so that $\sigma$ is $L U B$ at the unique solution of $\sigma$.

Example 3. $f$ finite-valued, $\sigma$ SS and not $\boldsymbol{U B}$ : Let $n=1$ and $\sigma=$ $\left\{t x \geq-t^{2}-1, t \in \mathbb{R}_{+}\right\}$. Then $F=\{x \geq-2\}$ and

$$
f(x)= \begin{cases}\frac{x^{2}}{4}-1, & x \leq 0, \\ -1, & \text { otherwise. }\end{cases}
$$

Example 4. $\sigma$ LUB at any feasible point and SS, $f$ not finite-valued: Let $n=2, T=\mathbb{Z} \backslash\{0\}$ and

$$
\sigma=\left\{t x_{1} \geq-t-t^{2}, t \in \mathbb{Z}, t>0,-t x_{1} \geq 3 t, t \in \mathbb{Z}, t<0\right\} .
$$


It can be realized that $F=[-2,+\infty) \times \mathbb{R}$, that $0_{2}$ is $S S$, and that $W(\bar{x}, \gamma) \subset\{(1,0)\}$ for all $\bar{x} \in F$ and all $\gamma \in] 0,1[$. Thus, $\sigma$ is SS and LUB at all its feasible points. Nevertheless, in this case $f(x)=+\infty$ for any $x=\left(x_{1}, x_{2}\right)$ with $x_{1}<-3$.

\section{Implicit aCtive CONStRaints}

We associate with $\sigma=\left\{a_{t}^{\prime} x \geq b_{t}, t \in T\right\}$ its normalized system $\widetilde{\sigma}=\left\{{\widetilde{a_{t}}}^{\prime} x \geq \widetilde{b_{t}}, t \in T\right\}$ defined by

$$
\left(\widetilde{a_{t}}, \widetilde{b_{t}}\right)= \begin{cases}\left\|a_{t}\right\|^{-1}\left(a_{t}, b_{t}\right), & \text { if } a_{t} \neq 0_{n}, \\ \left(a_{t}, b_{t}\right), & \text { otherwise. }\end{cases}
$$

Obviously, $\widetilde{\sigma}$ is a UB linear representation of $F$. Given $\bar{x} \in F$, we represent by $\widetilde{W}(\bar{x}, \gamma)$ and by $\widetilde{A}(\bar{x})$ the sets of $\gamma$-active constraints and the set of implicit active constraints at $\bar{x}$ relative to $\widetilde{\sigma}$, respectively. From the definition of the set of $\gamma$-active constraints we get its invariance under normalization in the sense that $a_{t} \in W(\bar{x}, \gamma)$ if and only if $\widetilde{a_{t}} \in \widetilde{W}(\bar{x}, \gamma)$. The next result compares the set of implicit active constraints at $\bar{x}$ relative to $\sigma$ and $\widetilde{\sigma}$.

Lemma 1. If $a \in A(\bar{x}) \backslash\left\{0_{n}\right\}$, then $\widetilde{a}:=\|a\|^{-1} a \in \widetilde{A}(\bar{x})$. Conversely, if $\sigma$ is LUB at $\bar{x}$ and $\widetilde{a} \in \widetilde{A}(\bar{x}) \backslash\left\{0_{n}\right\}$, then there exists $a \in A(\bar{x}) \backslash\left\{0_{n}\right\}$ such that $a=\|a\| \widetilde{a}$. Thus, cone $A(\bar{x}) \subset$ cone $\widetilde{A}(\bar{x})$ and the equality holds whenever $\sigma$ is LUB at $\bar{x}$.

Proof: If $a \in A(\bar{x}) \backslash\left\{0_{n}\right\}$, take a sequence $\left\{t_{k}\right\} \subset T$ such that $a_{t_{k}} \rightarrow a$ and $b_{t_{k}} \rightarrow a^{\prime} \bar{x}$. Without loss of generality (w.l.o.g.) we may assume that $a_{t_{k}} \neq 0_{n}$ for all $k$, hence $\widetilde{a_{t_{k}}} \rightarrow \widetilde{a}$ and $\widetilde{b_{t_{k}}} \rightarrow \widetilde{a} \bar{x}$, so $\widetilde{a} \in \widetilde{A}(\bar{x})$.

Now we take $\widetilde{a} \in \widetilde{A}(\bar{x}) \backslash\left\{0_{n}\right\}$. Let $\gamma$ and $\mu$ be positive numbers such that $W(\bar{x}, \gamma) \subset B\left(0_{n}, \mu\right)$. Let $\left\{t_{k}\right\} \subset T$ such that $\widetilde{a_{t_{k}}} \neq 0_{n}, \widetilde{a_{t_{k}}} \rightarrow \widetilde{a}$, and $\widetilde{b_{t_{k}}} \rightarrow \widetilde{a}^{\prime} \bar{x}$. Multiplying by $\bar{x}$ and by -1 both sequences, and summing up them, we get $\left(\widetilde{a_{t_{k}}} \bar{x}-\widetilde{b_{t_{k}}}\right) \rightarrow 0$. W.l.o.g. we may assume that $\widetilde{a_{t_{k}}}{ }^{\prime} \bar{x}-\widetilde{b_{t_{k}}}<\gamma$ for all $k$, so that $a_{t_{k}}^{\prime} \bar{x}-b_{t_{k}}<\gamma\left\|a_{t_{k}}\right\|$, so (2.1) gives $a_{t_{k}} \in W(\bar{x}, \gamma) \subset B\left(0_{n}, \mu\right)$, for all $k$. Thus we can assume, by passing to a subsequence if necessary, that $\left\{a_{t_{k}}\right\}$ is a convergent sequence. Let $a_{t_{k}} \rightarrow a \in \mathbb{R}^{n}$, in which case $\left\|a_{t_{k}}\right\| \rightarrow\|a\|$ and so $a=\|a\| \widetilde{a}$ because $\frac{a_{t_{k}}}{\left\|a_{t_{k}}\right\|}=\widetilde{a_{t_{k}}} \rightarrow \widetilde{a}$.

From $\frac{b_{t_{k}}}{\left\|a_{t_{k}}\right\|}=\widetilde{b_{t_{k}}} \rightarrow \widetilde{a}^{\prime} \bar{x} \quad$ we get that $b_{t_{k}} \rightarrow\|a\| \widetilde{a}^{\prime} \bar{x}$. Hence, $a=\|a\| \widetilde{a} \in$ $A(\bar{x})$.

Remark 1. Notice that $0_{n} \in \widetilde{A}(\bar{x})$ implies that $0_{n} \in A(\bar{x})$, while the converse does not hold.

Example 5. If $\sigma=\left\{t^{2} x \geq-t, t=1,2, \ldots\right\}$, then $D=\left\{\left(t^{2},-t\right), t=1,2, \ldots\right\}=$ $\operatorname{cl} D$ and $\widetilde{D}=\left\{\left(1,-t^{-1}\right), t=1,2, \ldots\right\}, \operatorname{cl} \widetilde{D}=\widetilde{D} \cup\{(1,0)\}$. Now, for $\bar{x}=0 \in$ $F=\mathbb{R}_{+}$, we have $A(\bar{x})=\emptyset$ and $\widetilde{A}(\bar{x})=\{1\}$, so that cone $A(\bar{x}) \neq$ cone $\widetilde{A}(\bar{x})$. The reason is that $\sigma$ is not LUB at $\bar{x}$. Observe that $\operatorname{cl}\left\{\widetilde{a_{t}}: t \in T(\bar{x})\right\}=\emptyset \varsubsetneqq \widetilde{A}(\bar{x})$.

In general, the SS property is neither transmitted from $\sigma$ to $\widetilde{\sigma}$ nor from $\widetilde{\sigma}$ to $\sigma$. In fact, $\sigma=\{-1 \leq t x \leq 1, t \in \mathbb{N}\}$, which is not LUB at its unique solution satisfies the SS condition whereas $\widetilde{\sigma}=\left\{-\frac{1}{t} \leq x \leq \frac{1}{t}, t \in \mathbb{N}\right\}$ does not (the same happens in Example 7, where the given system is highly stable whereas its normalized system is unstable). Analogously, the SS condition fails at the UB system $\left.\left.\sigma=\left\{t x \geq-t^{2}, t \in\right] 0,1\right]\right\}$ whereas $\widetilde{\sigma}$ is SS.

Lemma 2. Let $\sigma$ be LUB at some feasible point. If $\sigma$ is SS, then $\widetilde{\sigma}$ is SS. 
Proof: Let $\bar{x}, \widehat{x} \in F$ and let $\gamma, \mu, \varepsilon$ be positive scalars such that $W(\bar{x}, \gamma) \subset$ $B\left(0_{n}, \mu\right)$ and $a_{t}^{\prime} \widehat{x} \geq b_{t}+\varepsilon$ for all $t \in T$. We shall prove that $y:=\frac{\bar{x}+\widehat{x}}{2}$ is an SS point for $\tilde{\sigma}$. We discuss two possible cases for $t \in T$ such that $a_{t} \neq 0_{n}$ (the case $a_{t}=0_{n}$ is trivial).

If $a_{t}^{\prime} \bar{x}-b_{t} \geq \gamma\left\|a_{t}\right\|$, then ${\widetilde{a_{t}}}^{\prime} \bar{x} \geq \widetilde{b_{t}}+\gamma$ and ${\widetilde{a_{t}}}^{\prime} \widehat{x} \geq \widetilde{b_{t}}$, so that

$$
{\widetilde{a_{t}}}^{\prime} y \geq \widetilde{b_{t}}+\frac{\gamma}{2}
$$

Alternatively, if $a_{t}^{\prime} \bar{x}-b_{t}<\gamma\left\|a_{t}\right\|$, then $a_{t} \in W(\bar{x}, \gamma)$ and so $\left\|a_{t}\right\|^{-1} \geq \frac{1}{\mu}$. Since $a_{t}^{\prime} \bar{x} \geq b_{t}$ and $a_{t}^{\prime} \widehat{x} \geq b_{t}+\varepsilon$, we have

$$
{\widetilde{a_{t}}}^{\prime} y \geq \widetilde{b_{t}}+\frac{\varepsilon}{2\left\|a_{t}\right\|} \geq \widetilde{b_{t}}+\frac{\varepsilon}{2 \mu} .
$$

Combining (3.1) and (3.2) we get the aimed conclusion.

The next result expresses the polar of the feasible directions cone at a point $\bar{x} \in F, D(F ; \bar{x})^{0}$, in terms of $A(\bar{x})$. This expression will play a crucial role in Section 5 as far as $\bar{x}$ is an optimal solution of $P$ if and only if $c \in D(F ; \bar{x})^{0}$, and it is a strongly unique optimal solution if and only if $c \in \operatorname{int} D(F ; \bar{x})^{0}$. Notice that cone $A(\bar{x})$ is always a subset of $D(F, \bar{x})^{0}$, which follows immediately from the definition of $A(\bar{x})$.

Proposition 3. If $\sigma$ is $S S$ and $L U B$ at $\bar{x}$, then $0_{n} \notin \operatorname{conv} A(\bar{x})$, and cone $A(\bar{x})=$ $D(F, \bar{x})^{0}$ is pointed.

Proof: We show the first statement assuming the contrary, i.e., that $0_{n} \in$ conv $A(\bar{x})$. Let $d_{1}, \ldots, d_{m} \in A(\bar{x})$ and non-negative scalars $\lambda_{1}, \ldots, \lambda_{m}$ such that $\sum_{\left\{t_{i}^{r}\right\}_{r=1}^{\infty}{ }_{i=1}^{m} \subset T \text { such that }}^{m} \lambda_{i} d_{i}=0_{n}$ and $\sum_{i=1}^{m} \lambda_{i}=1$. For each $i=1, \ldots, m$ there exists a sequence

$$
\left(\begin{array}{c}
d_{i} \\
d_{i}^{\prime} \bar{x}
\end{array}\right)=\lim _{r}\left(\begin{array}{c}
a_{t_{i}^{r}} \\
b_{t_{i}^{r}}
\end{array}\right)
$$

Let $\widehat{x} \in F$ and $\varepsilon>0$ be such that $a_{t}^{\prime} \widehat{x} \geq b_{t}+\varepsilon$ for all $t \in T$. Multiplying by $(\widehat{x},-1)$ both members of $(3.3)$ we get $d_{i}^{\prime}(\widehat{x}-\bar{x})=\lim _{r}\left(a_{t_{i}^{r}}^{\prime} \widehat{x}-b_{t_{i}^{r}}\right) \geq \varepsilon$, whereas $0=\sum_{i=1}^{m} \lambda_{i} d_{i}^{\prime}(\widehat{x}-\bar{x}) \geq \sum_{i=1}^{m} \lambda_{i} \varepsilon=\varepsilon$. This is a contradiction.

Now, since the normalized system $\widetilde{\sigma}$ is UB and SS, by Lemma 2 , cone $\widetilde{A}(\bar{x})$ is closed and pointed ([11, Lemma 2.6(c)]) and cone $A(\bar{x})=D(F, \bar{x})^{0}$ ([8, Proposition 5.4]). The conclusion follows from Lemma 1.

Proposition 4. If $\bar{x}$ is an $S S$ element of $\sigma$, then $A(\bar{x})=\emptyset$. The converse statement holds if $\sigma$ is $L U B$ at $\bar{x}$.

Proof: Assume that $a_{t}^{\prime} \bar{x} \geq b_{t}+\varepsilon$ for some positive $\varepsilon$ and for all $t \in T$. Then, there is no sequence $\left\{\left(a_{t_{r}}, b_{t_{r}}\right)\right\}$ such that $\lim _{r} a_{t_{r}}=a$ satisfies that $a^{\prime} \bar{x}=\lim _{r} b_{t_{r}}$; so $A(\bar{x})=\emptyset$.

Now suppose that $\sigma$ is LUB at $\bar{x}$ and $A(\bar{x})=\emptyset$. Then $\widetilde{\sigma}$ is a UB system with $\widetilde{A}(\bar{x})=\emptyset$ by Lemma 1 and Remark 1 . Thus $\bar{x}$ is an SS point of $\widetilde{\sigma}$, by [11, Lemma $2.6(\mathrm{a})$ ], but we cannot conclude that $\bar{x}$ is an SS point of $\sigma$. So, we will consider the following new re-scaling of $\sigma$. Put

$$
\left(\widehat{a_{t}}, \widehat{b_{t}}\right)= \begin{cases}\left\|a_{t}\right\|^{-1}\left(a_{t}, b_{t}\right), & \text { if }\left\|a_{t}\right\|>1 \\ \left(a_{t}, b_{t}\right), & \text { otherwise }\end{cases}
$$


and consider the new UB system $\widehat{\sigma}=\left\{{\widehat{a_{t}}}^{\prime} x \geq \widehat{b_{t}}, t \in T\right\}$. Clearly $\widehat{F}=F$ and any SS point of $\widehat{\sigma}$ is also an SS point of $\sigma$. Moreover, $\widehat{A}(\bar{x})=\emptyset$; in fact assume that $\widehat{A}(\bar{x}) \neq \emptyset$ and let $u \in \widehat{A}(\bar{x})$. Then, there exists a sequence $\left\{a_{t_{k}}\right\}$ such that $\widehat{a_{t_{k}}} \rightarrow u$ and $\widehat{b_{t_{k}}} \rightarrow u^{\prime} \bar{x}$. Now, if $\left\|a_{t_{k}}\right\|>1$ for infinitely many $k^{\prime}$ s, then $u \in \widetilde{A}(\bar{x})=\emptyset$, which is a contradiction; if $\left\|a_{t_{k}}\right\| \leq 1$ for infinitely many $k^{\prime}$ s, then $u \in A(\bar{x})=\emptyset$, another contradiction. Thus $\widehat{A}(\bar{x})=\emptyset$, and hence [11, Lemma 2.6(a)] applies again to give that $\bar{x}$ is an SS point of $\widehat{\sigma}$. Therefore, $\bar{x}$ is also SS for $\sigma$.

\section{Geometric applications}

The following example shows a variety of possibilities that we have to deal with in the semi-infinite setting, even for a system that satisfies the strong Slater condition.

Example 6. Consider $\sigma=\left\{(2-k) x_{1}+(1-2 k) x_{2} \geq 1-k, k \in \mathbb{N}\right\}$. Then $\sigma$ is $S S$, and we have $F=\left\{\left(\begin{array}{c}x_{1} \\ x_{2}\end{array}\right): x_{2} \leq x_{1}, x_{2} \leq \frac{1}{2}-\frac{1}{2} x_{1}\right\}$. Observe that for $\gamma>0$,

$$
\begin{aligned}
\bar{x}^{1} \in \text { bd } F & :\left(\begin{array}{l}
1 / 3 \\
1 / 3
\end{array}\right) \\
W\left(\bar{x}^{1}, \gamma\right)=A\left(\bar{x}^{1}\right) & \left.:\left\{\begin{array}{l}
2-k \\
1-2 k
\end{array}\right): k \in \mathbb{N}\right\} \\
\text { cone } A\left(\bar{x}^{1}\right) & \left.:\left\{\begin{array}{l}
x_{1} \\
x_{2}
\end{array}\right): x_{2} \leq \frac{1}{2} x_{1}, x_{2}<2 x_{1}\right\} \cup\left\{0_{2}\right\} \\
D\left(F, \bar{x}^{1}\right)^{0} & \left.:\left\{\begin{array}{l}
x_{1} \\
x_{2}
\end{array}\right): x_{2} \leq-x_{1}, x_{2} \leq 2 x_{1}\right\}, \\
\bar{x}^{2} \in \text { bd } F & :\left(\begin{array}{l}
1 \\
0
\end{array}\right) \\
W\left(\bar{x}^{2}, \gamma\right) & :\left\{\left(\begin{array}{l}
2-k \\
1-2 k
\end{array}\right): k \in \mathbb{N} \text { large enough }\right\} \\
A\left(\bar{x}^{2}\right) & : \emptyset \\
\operatorname{cone} A\left(\bar{x}^{2}\right) & :\left\{0_{2}\right\} \\
D\left(F, \bar{x}^{2}\right)^{0} & :\left\{\left(\begin{array}{l}
-x \\
-2 x
\end{array}\right): x \geq 0\right\},
\end{aligned}
$$

and

$$
\begin{array}{lll}
\bar{x}^{3} \in \text { bd } F & : & 0_{2} \\
W\left(\bar{x}^{3}, \gamma\right)=A\left(\bar{x}^{3}\right) & : & \left(\begin{array}{c}
1 \\
-1
\end{array}\right) \\
\text { cone } A\left(\bar{x}^{3}\right)=D\left(F, \bar{x}^{3}\right)^{0} & : & \left\{\left(\begin{array}{c}
x \\
-x
\end{array}\right): x \geq 0\right\},
\end{array}
$$

for $\gamma>0$ small enough (exactly, $\gamma<\inf \left\{\frac{k-1}{\sqrt{(2-k)^{2}+(1-2 k)^{2}}}, k \in \mathbb{N}\right\}$ ).

Notice that $\sigma$ is not $U B$, but it is $L U B$ at $\bar{x}^{3}$. Furthermore, the interior of $F$ is not the set of (strong) Slater points; actually any boundary point of the form $\left(s, \frac{1}{2}-\frac{1}{2} s\right)$ is $S S$ for $s>\frac{1}{3}$. This is the case of $\bar{x}^{1}$ and $\bar{x}^{2}$. Moreover, $\bar{x}^{1} \in \operatorname{bd} F$ and $F$ is a full dimensional polyhedral convex set although $A\left(\bar{x}^{1}\right)$ is not compact and cone $A\left(\bar{x}^{1}\right)$ is not even closed; $\sigma$ is not $L U B$ at $\bar{x}^{1}$.

The LUB condition is related to the full dimensionality of $F$ as the following proposition shows.

Proposition 5. If $\operatorname{dim} F=n$, then $\sigma$ is LUB at some feasible point. Conversely, if $\sigma$ is $S S$ and $L U B$ at some feasible point, then $\operatorname{dim} F=n$.

Proof: Taking $\bar{x}$ and $\gamma>0$ such that $B(\bar{x}, \gamma) \subset F$ we have $W(\bar{x}, \gamma) \subset\left\{0_{n}\right\}$. Conversely, let $\sigma$ be SS and LUB at $\bar{x} \in F$. By Lemma 2 , the normalized system $\widetilde{\sigma}$ is SS, which gives that int $F \neq \emptyset$ by Propositions 1 and 2 (i). Therefore $\operatorname{dim} F=n$. 
In general, it is not easy to classify a given $\bar{x} \in F$ as interior or boundary point (specially when the SS condition fails). We have seen in Proposition 1 that, if $\sigma$ is SS and $\operatorname{dim} F=n$, then

$$
\operatorname{int} F=\left\{x \in \mathbb{R}^{n}: f(x)<0\right\}=\left\{x \in \mathbb{R}^{n}: \inf _{t \in T}\left(a_{t}^{\prime} x-b_{t}\right)>0\right\} .
$$

The system $\sigma=\left\{x \geq-\frac{1}{t}, t \in \mathbb{N}\right\}$ shows that we can have

$$
\operatorname{int} F \neq\left\{x \in \mathbb{R}^{n}: a_{t}^{\prime} x>b_{t}, t \in T\right\}
$$

even though $\operatorname{dim} F=n$ and $\sigma$ is UB and $\mathrm{SS}(0 \in \operatorname{bd} F$ is a Slater point of $\sigma)$. Proposition 1 shows that the finiteness of the supremum function $f$ is a sufficient condition to assure that any SS element is an interior point, and vice versa. This is also the case for UB systems, which can be seen by a straightforward calculation, or by an application of Proposition 2 (i) combined with the mentioned Proposition 1. Example 6 shows that we can not replace the UB condition by the LUB one. Obviously, another sufficient condition for $\bar{x} \in F$ to be an interior point of $F$ is that $\bar{x}$ is an SS point of $\widetilde{\sigma}$.

The next result provides two necessary conditions for a feasible point to be a boundary point of $F$. These two conditions extend [8, Proposition 5.1] and [11, Lemma 2.6(a)] from UB to systems which are LUB at some feasible point, respectively; the second one eliminates the unnecessary assumption that $\sigma$ is SS. We will make use of the following double inclusion ([8, Lemma 5.2]):

$$
A(\bar{x}) \backslash\left\{0_{n}\right\} \subset \bigcap_{\gamma>0} \operatorname{cl} W(\bar{x}, \gamma) \subset A(\bar{x}) \text { for all } \bar{x} \in F .
$$

Proposition 6. If $\sigma$ is $L U B$ at $\bar{x} \in \operatorname{bd} F$, then $\bigcap_{\gamma>0} \operatorname{cl} W(\bar{x}, \gamma) \neq \emptyset$ and $A(\bar{x})$ is a nonempty compact set.

Proof: Since the normalized system $\widetilde{\sigma}$ is UB at $\bar{x} \in$ bd $F$, Proposition 5.1 in [8] gives the existence of $u \in \bigcap_{\gamma>0} \operatorname{cl} \widetilde{W}(\bar{x}, \gamma)$. In the case that $u=0_{n}$, we necessarily have that $0_{n} \in \widetilde{W}(\bar{x}, \gamma)$ for all $\gamma>0$, which only occurs when $0_{n} \in W(\bar{x}, \gamma)$ for all $\gamma>0$, thus $0_{n} \in \bigcap_{\gamma>0} \operatorname{cl} W(\bar{x}, \gamma) \subset A(\bar{x})$, by (4.1). Then, $\bigcap_{\gamma>0} \operatorname{cl} W(\bar{x}, \gamma) \neq \emptyset$ and $A(\bar{x}) \neq \emptyset$. Suppose now that $u \neq 0_{n}$. From (4.1) applied to $\widetilde{\sigma}$, we get that

$$
\bigcap_{\gamma>0} \operatorname{cl} \widetilde{W}(\bar{x}, \gamma) \subset \widetilde{A}(\bar{x}),
$$

so $0_{n} \neq u \in \widetilde{A}(\bar{x})$. From Lemma 1 , it follows that $A(\bar{x}) \backslash\left\{0_{n}\right\} \neq \emptyset$. Finally, (4.1) gives that

$$
\emptyset \neq A(\bar{x}) \backslash\left\{0_{n}\right\} \subset \bigcap_{\gamma>0} \operatorname{cl} W(\bar{x}, \gamma),
$$

which, together with the LUB property of $\sigma$ at $\bar{x}$, implies that $A(\bar{x})$ is bounded, so it is compact because it is always a closed subset of $\mathbb{R}^{n}$.

Proposition 3 provides assumptions under which $D(F, \bar{x})^{0}$ can be replaced with cone $A(\bar{x})$, which depends on the data (instead of depending on the usually unknown set $F$ ). This allows us to obtain geometric information on $F$; for instance, a necessary condition for $F$ being quasipolyhedral (i.e., the non-empty intersections of $F$ with polytopes are polytopes).

Proposition 7. Let $\sigma$ be $S S$ and suppose that it is $L U B$ at $\bar{x} \in F$. Then, a necessary condition for $F$ to be quasipolyhedral is that cone $A(\bar{x})$ is polyhedral. 
Proof: Proposition 3 gives the equality cone $A(\bar{x})=D(F, \bar{x})^{0}$. If $F$ is quasipolyhedral, then $D(F, \bar{x})^{0}$ is polyhedral, so cone $A(\bar{x})$ must be polyhedral.

\section{Applications to Optimality CONDITIONS AND STABILITY}

The next two results provide optimality and strongly unique optimality tests of Karush-Kuhn-Tucker type. The first one shows that the combination of the SS and the LUB properties provides a constraint qualification in LSIP.

Theorem 1. Let $\bar{x} \in F$. If $c \in$ cone $A(\bar{x})$, then $\bar{x} \in F^{*}$. The converse statement holds if $\sigma$ is $S S$, and $L U B$ at $\bar{x}$.

Proof: The direct statement is [8, Corollary 5.5].

Now, assuming that $\sigma$ is SS and LUB at $\bar{x}$, according to Proposition $3, \bar{x}$ is an optimal solution of $P$ if and only if $c \in D(F, \bar{x})^{0}=$ cone $A(\bar{x})$.

Taking $c=(-1,-2)$ and the SS (but not UB) system $\sigma$ in Example 6, we have $\bar{x}^{1}, \bar{x}^{2} \in F^{*}$ despite of $c \notin \operatorname{cone} A\left(\bar{x}^{i}\right)$ (which coincides here with the ordinary active cone at $\left.\bar{x}^{i}\right), i=1,2$. Thus, the local boundedness of $\sigma$ at the checked point is essential for the above optimality test. Concerning $\bar{x}^{3}$, where $\sigma$ is LUB, $c \notin$ cone $A\left(\bar{x}^{3}\right)$ entails $\bar{x}^{3} \notin F^{*}$.

Theorem 2. Let $\bar{x} \in F$. If $c \in$ int cone $A(\bar{x})$, then $\bar{x}$ is a strongly unique optimal solution of $P$. The converse statement holds if $\sigma$ is SS, and LUB at $\bar{x}$.

Proof: It is similar to the previous one.

Theorem 2 has potential application in the context of parametric LSIP, where it is assumed that all the data in the given problem $P$, i.e., the triple $\pi=(a, b, c)$ that we identify with $P$ from now on, can be perturbed. We associate with $\pi$ the set $\Pi$ (called space of parameters) of all the similar problems (i.e., LSIP problems with $n$ decision variables and index set $T$ ) obtained through arbitrary perturbations of the data of bounded size. We consider $\Pi$ equipped with the pseudometric of the uniform convergence, i.e., given two parameters $\pi_{1}=\left(a^{1}, b^{1}, c^{1}\right)$ and $\pi_{2}=\left(a^{2}, b^{2}, c^{2}\right)$,

$$
d\left(\pi_{1}, \pi_{2}\right):=\max \left\{\left\|c^{1}-c^{2}\right\|, \sup _{t \in T}\left\|\left(\begin{array}{c}
a_{t}^{1} \\
b_{t}^{1}
\end{array}\right)-\left(\begin{array}{c}
a_{t}^{2} \\
b_{t}^{2}
\end{array}\right)\right\|\right\} ;
$$

we use the same symbol to mark a perturbation of the nominal problem $\pi$ and its associated objects (for instance, $W_{1}(x, \gamma)$ and $A_{1}(x)$ denote the set of $\gamma$-active constraints and the implicit active set at $x \in F_{1}$ relative to $\pi_{1} \in \Pi$, respectively). We say that an LSIP problem $\pi$ is UB (or LUB at some feasible point) if its constraint system is so. It is easy to see that the subspace of UB problems form an open and closed subset of $\Pi$. It has been proved (in [10]) that the subset of UB problems with strongly unique optimal solution contains an open and dense subset of the subspace of UB problems. This generic result does not hold for the class of problems which are LUB at some point, but we shall show (in Theorem 3 below) that it is possible to identify open subsets of $\Pi$ formed by problems with strongly unique optimal solution. Unfortunately, re-scaling here is of limited use because a small perturbation of a particular constraint of $\sigma$ with small coefficients could provoke a large perturbation in the equivalent system.

As a corollary of the next lemma we obtain that the LUB property at some point is stable in the following sense: If $\pi$ is LUB at $\bar{x} \in F$, then there are open neighborhoods $V$ of $\pi$ and $U$ of $\bar{x}$, such that $\pi_{1}$ is LUB at $x_{1}$, for any $\pi_{1} \in V$ and any $x_{1} \in U \cap F_{1}$. 
Lemma 3. Let $\pi=(a, b, c), x \in \mathbb{R}^{n}$, and $\gamma>0$ be such that $W(x, \gamma)$ is bounded. Then there exists an open neighborhood $V$ of $\pi$ such that the sets $W_{1}\left(z, \frac{\gamma}{3}\right)$ are uniformly bounded for any $\pi_{1} \in V$ and $z \in B\left(x, \frac{\gamma}{3}\right)$.

Proof: Suppose the contrary. Then there exist sequences $\left\{\pi_{k}\right\},\left\{z_{k}\right\}$, and $\left\{a_{t_{k}}^{k}\right\}$ such that $\pi_{k} \rightarrow \pi, z_{k} \in B\left(x, \frac{\gamma}{3}\right)$ and $a_{t_{k}}^{k} \in W_{k}\left(z_{k}, \frac{\gamma}{3}\right)$ such that $\left\|a_{t_{k}}^{k}\right\|>k, k=$ $1,2, \ldots$. For every $k \in \mathbb{N}$, we can find $y_{k} \in B\left(z_{k}, \frac{\gamma}{3}\right) \subset B\left(x, \frac{2 \gamma}{3}\right)$ such that $\left(a_{t_{k}}^{k}\right)^{\prime} y_{k}=$ $b_{t_{k}}^{k}$. Since $\left\{y_{k}\right\}$ is a bounded sequence, we may assume without loss of generality that $y_{k} \rightarrow y \in \operatorname{cl} B\left(x, \frac{2 \gamma}{3}\right)$ and $d\left(\pi_{k}, \pi\right)<\frac{1}{k}, k=1,2, \ldots$.

For $k=1,2, \ldots$, we have

whereby

$$
\left\|a_{t_{k}}^{k}\right\|-\left\|a_{t_{k}}\right\| \leq\|\| a_{t_{k}}^{k}\|-\| a_{t_{k}} \| \mid<\frac{1}{k}
$$

Let $k \in \mathbb{N}, k>1$. Then,

$$
\left\|a_{t_{k}}\right\| \geq\left\|a_{t_{k}}^{k}\right\|-\frac{1}{k}>k-\frac{1}{k}
$$

$$
\begin{aligned}
\left|a_{t_{k}}^{\prime} y_{k}-b_{t_{k}}\right| & \leq\left|\left(a_{t_{k}}-a_{t_{k}}^{k}\right)^{\prime} y_{k}\right|+\left|b_{t_{k}}^{k}-b_{t_{k}}\right| \\
& <\left\|a_{t_{k}}-a_{t_{k}}^{k}\right\|\left\|y_{k}\right\|+\frac{1}{k} \\
& <\frac{1}{k}\left(\left\|y_{k}\right\|+1\right) .
\end{aligned}
$$

Choose $\varepsilon_{k}$ such that $a_{t_{k}}^{\prime} y_{k}-b_{t_{k}}=-\varepsilon_{k}\left\|a_{t_{k}}\right\|$, then

$$
\left|\varepsilon_{k}\right|=\left|\frac{a_{t_{k}}^{\prime} y_{k}-b_{t_{k}}}{\left\|a_{t_{k}}\right\|}\right|<\frac{\frac{1}{k}\left(\left\|y_{k}\right\|+1\right)}{k-\frac{1}{k}}=\frac{\left\|y_{k}\right\|+1}{k^{2}-1} .
$$

From the above inequality, for $k$ sufficiently large, we obtain

$$
\left|\varepsilon_{k}\right|<\frac{\gamma}{3}
$$

In that case, $y_{k}+\varepsilon_{k} \frac{a_{t_{k}}}{\left\|a_{t_{k}}\right\|} \in B(x, \gamma)$, because $\left\|y_{k}-x\right\| \leq \frac{2 \gamma}{3}$. Moreover,

$$
a_{t_{k}}^{\prime}\left(y_{k}+\varepsilon_{k} \frac{a_{t_{k}}}{\left\|a_{t_{k}}\right\|}\right)=a_{t_{k}}^{\prime} y_{k}+\varepsilon_{k}\left\|a_{t_{k}}\right\|=b_{t_{k}},
$$

so $a_{t_{k}} \in W(x, \gamma)$ (a bounded set). This contradicts $\left\|a_{t_{k}}\right\|>k-\frac{1}{k}$ for all $k \in \mathbb{N}$.

We consider the following sets of parameters:

$\Pi_{S}=\{\pi \in \Pi: \pi$ has an optimal solution $\}$,

$\Pi_{S U}=\{\pi \in \Pi: \pi$ has a strongly unique optimal solution $\}$.

According to [7, Theorem 3.1], if the constraint system of a problem $\pi$ is SS, then it is stable with respect to consistency, i.e., there is some neighborhood of $\pi$ such that any of the problems in that neighborhood is consistent. Other stability properties are related to the set-valued optimal mapping $\mathcal{F}^{*}: \Pi \rightrightarrows \mathbb{R}^{n}$ that assigns to each problem $\pi_{1}$ its optimal solution set, $F_{1}^{*}$, and to the set-valued mapping of implicit constraints $\mathcal{A}: \Pi \times \mathbb{R}^{n} \rightarrow \mathbb{R}^{n}$, such that $\mathcal{A}\left(\pi_{1}, x\right)$ is the set defined as in (1.3) for all $x \in \mathbb{R}^{n}$, with $D_{1}$ as in (1.4); this mapping is totally different to the so-called active set mapping from $\mathbb{R}^{n}$ to $\mathbb{R}^{n}$ considered in [5], which consists of the intersection of the convex cone generated by the ordinary active constraints with the unit sphere, with fixed data. The following continuity concepts are due to Bouligand and Kuratowski (see [1] or [2] for a general setting). $\mathcal{F}^{*}$ is said to be lower semicontinuous at $\pi \in \Pi$ (lsc in brief) if, for each open set $U \subset \mathbb{R}^{n}$ 
such that $U \cap \mathcal{F}^{*}(\pi) \neq \emptyset$, there exists an open neighborhood $V \subset \Pi$ of $\pi$, such that $U \cap \mathcal{F}^{*}\left(\pi_{1}\right) \neq \emptyset$ for each $\pi_{1} \in V$. $\mathcal{F}^{*}$ is upper semicontinuous at $\pi \in \Pi$ (usc in brief) if, for each open set $U \subset \mathbb{R}^{n}$ such that $\mathcal{F}^{*}(\pi) \subset U$, there exists an open neighborhood $V \subset \Pi$ of $\pi$, such that $\mathcal{F}^{*}\left(\pi_{1}\right) \subset U$ for each $\pi_{1} \in V . \mathcal{F}^{*}$ is continuous at $\pi \in \Pi$ if it is lsc and usc $\pi \in \Pi$. Finally, $\mathcal{F}^{*}$ is closed at $\pi \in \operatorname{dom} \mathcal{F}^{*}$ if for any sequences $\left\{\pi_{k}\right\} \subset \mathbb{R}^{n}$ and $\left\{x_{k}\right\} \subset \mathbb{R}^{n}$ satisfying $x_{k} \in \mathcal{F}^{*}\left(\pi_{k}\right)$ for all $k \in \mathbb{N}$, $\lim _{k} \pi_{k}=\pi$, and $\lim _{k} x_{k}=x$, one has $x \in \mathcal{F}^{*}(\pi)$. It is well known that $\mathcal{F}^{*}$ is usc at $\pi \in \operatorname{dom} \mathcal{F}^{*}$ whenever $\mathcal{F}^{*}$ is closed and uniformly bounded at $\pi$ (i.e., there exists a bounded set $C \subset \mathbb{R}^{n}$ such that $\mathcal{F}^{*}\left(\pi_{1}\right) \subset C$ for any $\pi_{1}$ sufficiently close to $\pi)$. Similar definitions and properties take place for the mapping $\mathcal{A}$ to be lsc, usc, continuous, closed, or uniformly bounded at $(\pi, x)$, where we consider the product topology on $\Pi \times \mathbb{R}^{n}$.

We state the following proposition for further reference (its proof can be found in $[6$, Theorem 10.4]).

Proposition 8. Let $\pi \in \Pi_{S}$ and assume that $\sigma$ is SS.

(i) If $F^{*}$ is a bounded set, then $\mathcal{F}^{*}$ is usc at $\pi$.

(ii) If $F^{*}$ is a singleton, then $\mathcal{F}^{*}$ is lsc at $\pi$.

Proposition 9. $\mathcal{A}$ is closed at any $(\pi, x) \in \Pi \times \mathbb{R}^{n}$.

Proof: Let us take an arbitrary $(\pi, x)$ and sequences $\left\{\left(\pi_{k}, x_{k}\right)\right\} \subset \Pi \times \mathbb{R}^{n}$ and $\left\{a_{k}\right\} \subset \mathbb{R}^{n}$ such that $a_{k} \in \mathcal{A}\left(\pi_{k}, x_{k}\right)$ for all $k \in \mathbb{N},\left(\pi_{k}, x_{k}\right) \rightarrow(\pi, x)$, and $a_{k} \rightarrow a$. From the definition of the mapping $\mathcal{A}$, for each $k \in \mathbb{N}$, we can find $t_{k} \in T$ such that

$$
\left\|a_{k}-a_{t_{k}}^{k}\right\|<\frac{1}{k} \text { and }\left|\left(a_{t_{k}}^{k}\right)^{\prime} x_{k}-b_{t_{k}}^{k}\right|<\frac{1}{k} .
$$

Let us fix $\varepsilon>0$ and $k_{0} \in \mathbb{N}$ such that $\left\|a_{k}-a\right\|<\varepsilon, \frac{1}{k}<\varepsilon$, and $d\left(\pi_{k}, \pi\right)<\varepsilon$ for every $k \geq k_{0}$. Then

$$
\left\|a-a_{t_{k}}^{k}\right\| \leq\left\|a-a_{k}\right\|+\left\|a_{k}-a_{t_{k}}^{k}\right\|<2 \varepsilon,
$$

and

for $k \geq k_{0}$. Thus $a_{t_{k}} \rightarrow a$. Moreover,

$$
\left\|a-a_{t_{k}}\right\| \leq\left\|a_{t_{k}}^{k}-a\right\|+\left\|a_{t_{k}}-a_{t_{k}}^{k}\right\|<3 \varepsilon,
$$

$$
a_{t_{k}}^{\prime} x-b_{t_{k}}=a_{t_{k}}^{\prime}\left(x-x_{k}\right)+\left(a_{t_{k}}-a_{t_{k}}^{k}\right)^{\prime} x_{k}+\left(a_{t_{k}}^{k}\right)^{\prime} x_{k}-b_{t_{k}}^{k}+b_{t_{k}}^{k}-b_{t_{k}} \rightarrow 0 .
$$

Hence

$$
a^{\prime} x=\lim _{k} a_{t_{k}}^{\prime} x=\lim _{k} b_{t_{k}} .
$$

Therefore $a \in \mathcal{A}(\pi, x)$, which proves the proposition.

Proposition 10. Let $\pi \in \Pi$ be LUB at $\bar{x} \in F$. Then $\mathcal{A}$ is usc at $(\pi, \bar{x})$.

Proof: Let $\gamma>0$ be such that $W(\bar{x}, \gamma)$ is bounded. By Lemma 3, there exist an open set $V \subset \Pi$ and a bounded closed set $C \subset \mathbb{R}^{n}$ such that $\pi \in V$, and $W_{1}\left(x, \frac{\gamma}{3}\right) \subset C$ for any $\pi_{1} \in V$ and $x \in B\left(\bar{x}, \frac{\gamma}{3}\right)$. From (4.1),

$$
\mathcal{A}\left(\pi_{1}, x\right)=A_{1}(x) \subset \bigcap_{\gamma^{\prime}>0} \operatorname{cl} W_{1}\left(x, \gamma^{\prime}\right) \cup\left\{0_{n}\right\} \subset \operatorname{cl} W_{1}\left(x, \frac{\gamma}{3}\right) \cup\left\{0_{n}\right\} \subset C \cup\left\{0_{n}\right\},
$$

for all $\left(\pi_{1}, x\right), \pi_{1} \in V, x \in B\left(\bar{x}, \frac{\bar{\gamma}}{3}\right)$. So, $\mathcal{A}$ is uniformly bounded at $(\pi, \bar{x})$. This together with the closedness of $\mathcal{A}$ at $(\pi, \bar{x})$ established in Proposition 9 above proves that $\mathcal{A}$ is usc at $(\pi, \bar{x})$.

Definition. $\pi \in \Pi$ satisfies the Extended Nürnberger (EN in brief) condition at $\bar{x} \in F$ if there exists $\left\{\bar{d}_{1}, \ldots, \bar{d}_{n}\right\} \subset A(\bar{x})$ such that $c \in$ cone $\left\{\bar{d}_{1}, \ldots, \bar{d}_{n}\right\}$ and $c \in$ int cone $\left\{d_{1}, \ldots, d_{n}\right\}$ for any set $\left\{d_{1}, \ldots, d_{n}\right\} \subset A(\bar{x})$ such that $c \in$ cone $\left\{d_{1}, \ldots, d_{n}\right\}$. We say that $\pi \in \Pi$ satisfies the Extended Nürnberger condition if $\pi$ satisfies this condition at some $\bar{x} \in F$. 
Proposition 11. If $\pi$ satisfies the Extended Nürnberger condition at some point $\bar{x}$ of $F$, then $\pi \in \Pi_{S U}$ and $F^{*}=\{\bar{x}\}$. If in addition $\sigma$ is $S S$, then $\mathcal{F}^{*}$ is continuous at $\pi$ and $\pi \in \operatorname{int} \Pi_{S}$.

Proof: The fact that $A(\bar{x}) \subset D(F, \bar{x})^{0}$, under the EN condition, implies that $\pi \in \Pi_{S U}$ and $F^{*}=\{\bar{x}\}$. Moreover, if $\sigma$ is SS, Proposition 8 gives the continuity of $\mathcal{F}^{*}$ at $\pi$, so for any $\varepsilon>0$ there is some $\delta>0$ such that $\emptyset \neq F_{1}^{*} \subset B(\bar{x}, \varepsilon)$ whenever $d\left(\pi, \pi_{1}\right)<\delta$. Thus $\pi \in \operatorname{int} \Pi_{S}$.

The following theorem gives conditions for $\pi \in$ int $\Pi_{S U}$, involving the SS condition, the EN condition, and the LUB property.

Theorem 3. Let $\sigma$ be SS. If $\pi \in \Pi$ satisfies the Extended Nürnberger condition at $\bar{x} \in \mathrm{bd} F$ and $\pi$ is $L U B$ at $\bar{x}$, then $\pi \in \operatorname{int} \Pi_{S U}$. The converse statement holds if $\pi$ is $L U B$ at the unique solution $\bar{x} \in F^{*}$.

Proof: Since $\sigma$ is SS, the problem $\pi$ is stable with respect to consistency. Assume that $\pi \in \Pi$ satisfies the EN condition at $\bar{x} \in \operatorname{bd} F$ and $\pi$ is LUB at $\bar{x}$. Let us suppose that $\pi \notin \operatorname{int} \Pi_{S U}$. By the previous result $\pi \in \operatorname{int} \Pi_{S}$, therefore there exists a sequence $\left\{\pi_{k}\right\} \subset \Pi_{S} \backslash \Pi_{S U}$ such that $\pi_{k} \rightarrow \pi$. We may also assume that there is a sequence $\left\{x_{k}\right\}$ such that $x_{k} \in F_{k}^{*}, x_{k} \rightarrow \bar{x}$ (see Proposition 11 and its proof), and each $\pi_{k}$ is LUB at $x_{k}, k \in \mathbb{N}$. Let $A_{k}\left(x_{k}\right):=\mathcal{A}\left(\pi_{k}, x_{k}\right)$ denote the set of implicit active constraints of $\pi_{k}$ at $x_{k}, k \in \mathbb{N}$; similarly $A(\bar{x}):=\mathcal{A}(\pi, \bar{x})$. From Theorem 1 , for a given $k \in \mathbb{N}$ and $x_{k} \in F_{k}^{*}$ there exist $M \leq n, \beta_{k}^{i}>0$ and $a_{k}^{i} \in A_{k}\left(x_{k}\right)$, $i=1, \ldots, M$ such that the vectors $a_{k}^{i}, i=1, \ldots, M$ are linearly independent and

$$
c_{k}=\sum_{i=1}^{M} \beta_{k}^{i} a_{k}^{i} .
$$

Taking into account that $\pi_{k} \in \Pi_{S} \backslash \Pi_{S U}$, we get $M<n$. Without loss of generality, we may assume that for each $k \in \mathbb{N}$ we have the same $M \in \mathbb{N}$. Since the mapping of implicit constraints $\mathcal{A}$ is usc at $(\pi, \bar{x})$, whereby $a_{k}^{i} \rightarrow a_{i} \in A(\bar{x}), i=1, \ldots, M$. We shall prove that the sequences $\left\{\beta_{k}^{i}\right\}_{k \geq 1}, i=1, \ldots, M$ are bounded. Let us assume the contrary and take the sequence $\beta_{k}=\sum_{i=1}^{M} \beta_{k}^{i} \rightarrow \infty$. If in the equality (5.2) we divide by $\beta_{k}$ and take limit for $k \rightarrow \infty$, we obtain (by passing to a subsequence if necessary)

$$
0_{n}=\sum_{i=1}^{M} \theta^{i} a_{i}
$$

where $\sum_{i=1}^{M} \theta^{i}=1$, so $0_{n} \in \operatorname{conv} A(\bar{x})$. Proposition 3 , provides a contradiction. So we may assume w.l.o.g. that each sequence $\left\{\beta_{k}^{i}\right\}_{k}$ converges, say $\beta_{k}^{i} \rightarrow \beta_{i}$, $i=1, \ldots, M$. Hence $c=\sum_{i=1}^{M} \beta_{i} a_{i}$ with $M<n$, which means that $\pi$ does not satisfy the EN condition at $\bar{x}$. This is a contradiction.

For the converse, let us suppose that $\pi \in \operatorname{int} \Pi_{S U}$ and $\pi$ is LUB at the unique solution $\bar{x} \in F^{*}$. Let $\gamma>0$ be such that $R:=\sup \left\{\left\|a_{t}\right\|: a_{t} \in W(\bar{x}, \gamma)\right\}<\infty$. There exist $M \leq n, \alpha_{i}>0$ and $a_{i} \in A(\bar{x}), i=1, \ldots, M$, such that the vectors $a_{i}$, $i=1, \ldots, M$, are linearly independent and

$$
c=\sum_{i=1}^{M} \alpha_{i} a_{i} .
$$

Here

$$
a_{i}=\lim _{r} a_{t_{r}^{i}} \quad \text { and } \quad a_{i}^{\prime} \bar{x}=\lim _{r} b_{t_{r}^{i}}
$$


for some sequences $\left\{\left(a_{t_{r}^{i}}^{\prime}, b_{t_{r}^{i}}\right)\right\}_{r \geq 1}, i=1, \ldots, M$. Assume that $M<n$. So, there exists a non null vector $u \in \mathbb{R}^{n}$ such that $\|u\|=1$ and $a_{i}^{\prime} u=0$, and hence $\lim _{r} a_{t_{r}^{i}}^{\prime} u=a_{i}^{\prime} u=0, i=1, \ldots, M$. We shall construct a sequence $\left\{\pi_{k}\right\}$, $\pi_{k}=\left(a^{k}, b^{k}, c^{k}\right)$, such that $\pi_{k} \rightarrow \pi$, and $\bar{x}$ and $\bar{x}-\frac{1}{k} u \in F_{k}^{*}$, for $k \in \mathbb{N}$ large enough. For each $k \in \mathbb{N}$ we define

$$
\begin{gathered}
a_{t}^{k}:=a_{t}, \quad t \in T, \text { and } c^{k}:=c, \\
b_{t}^{k}= \begin{cases}b_{t}-\frac{1}{k}\left|a_{t}^{\prime} u\right|, & t \in\left\{t \in T: a_{t} \in W(\bar{x}, \gamma)\right\}, \\
b_{t}, & \text { otherwise. }\end{cases}
\end{gathered}
$$

Then

$$
d\left(\pi_{k}, \pi\right)=\sup _{t \in T}\left\{\left|b_{t}^{k}-b_{t}\right|\right\}=\sup _{t \in\left\{t \in T: a_{t} \in W(\bar{x}, \gamma)\right\}} \frac{1}{k}\left|a_{t}^{\prime} u\right| \leq \frac{1}{k} R\|u\|=\frac{R}{k},
$$

whereby $\pi_{k} \rightarrow \pi$. From the definition of $b_{t}^{k}$, and recalling that whenever $a_{t} \notin$ $W(\bar{x}, \gamma)$, we have that $a_{t}^{\prime} y \geq b_{t}$, for all $y \in B(\bar{x}, \gamma)$, for $k \in \mathbb{N}$ large enough and for any $t \in T$,

$$
\begin{aligned}
& a_{t}^{\prime} \bar{x} \geq b_{t} \geq b_{t}-\frac{1}{k}\left|a_{t}^{\prime} u\right|, \text { and } \\
& a_{t}^{\prime}\left(\bar{x}-\frac{1}{k} u\right) \geq b_{t}-\frac{1}{k}\left|a_{t}^{\prime} u\right|,
\end{aligned}
$$

therefore $\bar{x}$ and $\bar{x}-\frac{1}{k} u \in F_{k}, k \in \mathbb{N}$ large enough.

We have that, for each $i=1, \ldots, M$,

$$
\begin{gathered}
a_{i}^{\prime} \bar{x}=\lim _{r} b_{t_{r}^{i}}=\lim _{r} b_{t_{r}^{i}}^{k} \text { and } \\
a_{i}^{\prime}\left(\bar{x}-\frac{1}{k} u\right)=\lim _{r}\left(a_{t_{r}^{i}}^{\prime} \bar{x}-\frac{1}{k} a_{t_{r}^{\prime}}^{\prime} u\right)=\lim _{r} b_{t_{r}^{i}}=\lim _{k} b_{t_{r}^{i}}^{k} .
\end{gathered}
$$

These facts imply that for every $k \in \mathbb{N}$ large enough, $a_{i}$ is in $A_{k}(\bar{x})$ and in $A_{k}\left(\bar{x}-\frac{1}{k} u\right), i=1, \ldots, M$, hence $\bar{x}$ and $\bar{x}-\frac{1}{k} u \in F_{k}^{*}$, which contradicts the fact that $\pi \in \operatorname{int} \Pi_{S U}$. Therefore $M=n$.

The following example shows that there are problems $\pi \in \operatorname{int} \Pi_{S U}$ which are not LUB at the strongly unique optimal solution.

Example 7. Let $n=2$, and let $T$ be the set of non-null integers. Consider the following problem:

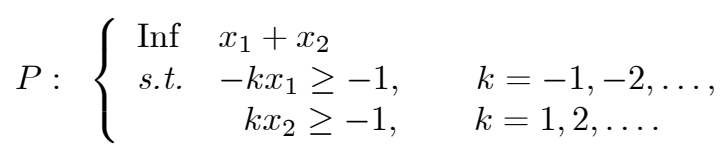

Then $F=\mathbb{R}_{+}^{2}, F^{*}=\left\{0_{2}\right\}$, and $W\left(0_{2}, \gamma\right)$ is unbounded for all $\gamma>0$. The set of active implicit constraints at $\bar{x}=0_{2}$ is empty. Nonetheless, $\pi \in \operatorname{int} \Pi_{S U}$ because $\bar{x}=0_{2}$ is a strongly unique optimal solution of $\pi$, and there is an open neighborhood of $\pi$ where the feasible set remains constant. Notice that this problem does not fulfill the $E N$ condition at $\bar{x}=0_{2}$.

Acknowledgement. The authors wish to thank the two anonymous referees for their valuable comments and suggestions. 


\section{REFERENCES}

[1] Aubin JP, Frankowska H (1990) Set-Valued Analysis, Birkhäuser, Boston.

[2] Bank B, Guddat J, Klatte D, Kummer B, Tammer K (1983) Non-Linear Parametric Optimization. Birkhäuser-Verlag, Basel.

[3] Cánovas MJ, Klatte D, López MA, Parra J (2007) Metric regularity in convex semi-infinite optimization under canonical perturbations. SIAM J. Optim. 18: 717-732.

[4] Dontchev AL, Zolezzi T (1993) Well-Posed Optimization Problems. Springer-Verlag, Berlin.

[5] Fajardo MD, López MA (1999) Locally Farkas-Minkowski Systems in Convex Semi-infinite Programming. J. Optim. Theor Appl. 103: 313-335.

[6] Goberna MA, López MA (1998) Linear Semi-Infinite Optimization. J. Wiley \& Sons, Chichester.

[7] Goberna MA, López MA, Todorov MI (1996) Stability theory for linear inequality systems. SIAM J. Matrix Anal. Appl. 17: 730-743.

[8] Goberna MA, López MA, Todorov MI (2003) Extended active constraints in linear optimization with applications. SIAM J. Optim. 14: 608-619.

[9] Goberna MA, López MA, Todorov MI (2003) A sup-function approach to linear semi-infinite optimization. Journal of Math. Sci. 116: 3359-3368.

[10] Goberna MA, López MA, Todorov MI (2003) A generic result in linear semi-infinite optimization. Appl. Math. Optim. 48: 181-193.

[11] Helbig, S, Todorov MI (1998) Unicity results for general linear semi-infinite optimization problems using a new concept of active constraints. Appl. Math. Optim. 38: 21-43.

[12] Hettich R, Zencke P (1982) Numerische Methoden der Approximation und semi-infiniten Optimierung. Teubner, Stuttgart.

[13] Lucchetti R (2006) Convexity and Well-Posed Problems. Springer-Verlag, New York.

[14] Nürnberger G (1985) Unicity in semi-infinite optimization, In Brosowski B, Deutsch (eds.), Parametric Optimization and Approximation. Birkhäuser, Basel, 231-247.

[15] Rockafellar RT (1970) Convex Analysis. Princeton UP, Princeton.

Dep. of Statistics and Operations Research, Alicante University, 03071 Alicante, Spain. E-Mail: mgoberna@ua.es. Research supported by micinn of Spain, Grant MTM2008-06695-C03-01.

Instituto de Física y Matemáticas, Universidad Tecnológica de Mixteca, Carretera a Acatlima Km.2.5 Huajuapan de Leon, Oaxaca, México. E-Mail: lanchoga@mixteco.utm.mx.

Dep. of Physics and Mathematics, Udla, 72820 San Andrés Cholula, Puebla, Mexico. On leave from IMI-BAS, Sofia, Bulgaria. E-Mail: maxim.todorov@udlap.mx. Research PARTIALlY SUPPORTED By CONACYT OF MX.GRANT 55681.

Facultad de Ciencias Económicas, Instituto de Ciencias Básicas, Universidad Nacional de Cuyo, 5500 Mendoza, Argentina. E-Mail: vvera@uncu.edu.ar. Research supported by SeCyt-UnCuyo of Argentina, Grant Res. 1094/09-R. (V.N. Vera de Serio. Corresponding aUthor) 